\title{
Associative matching and cumulative proactive inhibition*
}

\author{
BENTON J. UNDERWOOD, PAUL K. BRODER \\ and \\ JOEL ZIMMERMAN \\ Northwestern University, Evanston, Ill. 60201
}

\begin{abstract}
On each of 4 successive days, the S learned a different paired-associate list. Twenty-four hours after learning each list, retention was measured by an associative-matching test in which stimulus and response terms were provided. Associative-matching performance did not differ across the four lists, i.e., there was no cumulative proactive inhibition.
\end{abstract}

Cumulative proactive interference is shown in the recall of successively learned paired-associate lists. The $\mathrm{S}$ learns a list, recalls it, say, $24 \mathrm{~h}$ later, learns a second list, recalls it $24 \mathrm{~h}$ later, and so on. Recall is inversely related to the number of prior lists learned and recalled. The most extensive study reported (Keppel, Postman, \& Zavortink, 1968) involved 36 lists and a 48-h retention interval. Recall fell from $70 \%$ on the first list to $4 \%$ on the 36th list. Unpaced recall will reduce the decrement but not eliminate it (Underwood \& Ekstrand, 1967), as will several unreinforced recall trials (Keppel et al, 1968).

The question posed by the present experiment is whether or not cumulative proactive interference would be found using an associative-matching test of retention. In this test the $S$ is given the stimulus and response terms and is asked to pair them as they had been paired during the learning phase. As is apparent, the major analytical value of this test is that it eliminates response availability or response retrieval in the retention test. However, successful performance on the test does require that the associations between stimulus and response terms maintain their integrity.

\section{METHOD \\ Lists}

The $\mathrm{S}$ learned four lists, with the matching test on each list being given $24 \mathrm{~h}$ following learning. Each list consisted of 24 pairs of high-frequency (AA) two-syllable words taken from Thorndike and Lorge. The 192 words were paired randomly and assigned to the four lists randomly.

\section{Procedure and Ss}

The lists were presented at a 2.2 -sec rate and learning was carried to 20 correct anticipations on a single trial. The first list was learned on a Monday. On Tuesday, the associative-matching test was given, a second list learned, and so on. Hence, each $S$ served 5 consecutive days. On the matching test, the $S$ was given the stimulus terms in one column on a sheet of paper and the response terms in another column. He was asked to place the

*This research was supported by the Personnel and Training Research Programs, Psychological Sciences Division Office of Naval Research, under Contract No. N00014-67-A-0356-0010,

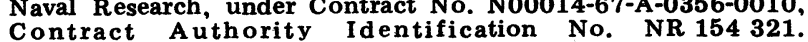
Reproduction in whole or in part is permitted for any purpose of the United States Government. number of the response term next to its appropriate stimulus term, guessing if necessary. This test was unpaced. The position of the four lists across the four stages was completely counterbalanced. The 24 Ss were college students enrolled in the course in introductory psychology.

\section{RESULTS}

The mean numbers of trials required to learn the lists to a criterion of 20 correct anticipations on a single trial were $13.75,10.21,10.00$, and 9.17 at each of the four successive stages. The learning-to-learn effect was highly reliable, $\mathrm{F}(3,69)=10.26, \mathrm{p}<.01$.

The mean numbers of pairs correctly matched at each successive stage were $23.33,23.08,23.04$, and 22.83 . Matching performance was higher than performance on the last trial of original learning, where (in attaining the criterion of 20 correct) the means varied between 20.54 and 21.25. The increase may have been due in part to correct guesses among the alternatives remaining when known pairings were completed. The matching means suggest a slight decrement across stages, but it is not statistically reliable $(F<1)$. This conclusion was not changed when loss scores were used, nor when projected scores were used to account for differences in rate at which the learning reached the criterion on the four successive lists. It must be concluded that retention as measured by an associative-matching procedure showed little evidence of cumulative proactive interference for these paired-associate lists.

\section{DISCUSSION}

If the matching procedure is accepted as a technique for measuring the integrity of an association, it is to be concluded that cumulative proactive interference does not result from an associative loss per se. It might be argued that the matching test requires very little associative strength for successful performance. Thus, even if the association had been severely weakened, it was sufficiently strong to provide appropriate matching. It would be expected (by this argument) that interference in a matching test would be observed after many, many stages of learning. There is no way to deny this possibility from the present data. However, when cumulative interference is found in recall, its magnitude of increase is greatest for the first few stages. If the interference occurs only because of weakened associations between the stimulus and response terms, some evidence should have been found for it on the matching tests of the present experiment. The evidence seems to indicate that cumulative proactive interference represents an interference in the retrieval of the appropriate response terms.

\section{REFERENCES}

Keppel, G., Postman, L., \& Zavortink, B. Studies of learning to learn: VIII. The influence of massive amounts of training upon the learning and retention of paired associate lists. Journal of Verbal Learning \& Verbal Behavior, 1968, 7, 790-796.

Underwood, B. J., \& Ekstrand, B. R. Word frequency and accumulative proactive inhibition. Journal of Experimental Psychology, 1967, 74, 193-198.

(Received for publication September 10, 1972.) 